\title{
A case-control study of the relationship between visceral fat and development of uterine fibroids
}

\author{
KEJUAN SUN ${ }^{1}$, YING XIE ${ }^{2}$, NA ZHAO ${ }^{3}$ and ZENGNING LI $^{2}$ \\ Departments of ${ }^{1}$ Nursing, ${ }^{2}$ Nutrition and ${ }^{3}$ Gynecology, The First Hospital of Hebei Medical University, \\ Shijiazhuang, Hebei 050031, P.R. China
}

Received January 7, 2019; Accepted April 18, 2019

DOI: $10.3892 / \mathrm{etm} .2019 .7575$

\begin{abstract}
Relationship between visceral fat and development of uterine fibroids in adult women was investigated. A total of 89 patients with uterine fibroids treated in the First Hospital of Hebei Medical University from April 2016 to April 2018 were enrolled. Another 81 healthy women without uterine fibroids receiving physical examination in the same period were selected as the control group. Self-designed questionnaires were used to investigate the general conditions of the subjects, bioelectrical impedance analysis was employed to analyze obesity-related indicators, and their correlation with the risk of uterine fibroids was explored. Visceral fat area (VFA), body mass index (BMI), body fat percentage, waist circumference, waist-height ratio and waist-hip ratio were positively correlated with the incidence rate of uterine fibroids, and the odds ratio (OR), 95\% confidence interval (CI) was $3.910(2.029,7.536)$, $2.716(1.444,5.110), 4.335$ (1.507, 12.469), 2.881 (1.531, 5.423), $3.837(1.914,7.692)$ and $7.707(3.501,16.965)$, respectively. VFA and body fat percentage were correlated with the size of uterine fibroids, but the correlations were relatively weak. With the increase in the VFA, BMI, waist circumference, waist-height ratio, and waist-hip ratio, the risk of uterine fibroids was elevated. The BMI, VFA and waist circumference of patients with uterine fibroids were gradually increased with increasing age, showing statistically significant differences. Increased body fat (especially abdominal visceral fat) is able to enhance the risk of uterine fibroids. For perimenopausal women, the waist-hip ratio measured can be used as an indicator screening high-risk groups of uterine fibroids. This method is simple, easy and inexpensive. At the same time, providing nutrition guidance and changing diet and exercise
\end{abstract}

Correspondence to: Dr Zengning Li, Department of Nutrition, The First Hospital of Hebei Medical University, 89 Donggang Road, Shijiazhuang, Hebei 050031, P.R. China

E-mail:mizu73@126.com; lizengning@126.com

Key words: bioelectrical impedance analysis, uterine fibroids, visceral fat, central obesity habits are important measures to prevent the development of uterine fibroids.

\section{Introduction}

Uterine fibroids, one of the most common benign tumors in the female reproductive organs, occur mostly among women aged over 30 years, and women of childbearing age are mainly affected. However, some studies have revealed that the incidence rate of uterine fibroids is also very high in perimenopausal women aged 40-54 years due to ovarian hormone imbalance and related hormone imbalance $(1,2)$. Common complications of uterine fibroids include anemia, osteoporosis and infertility, having long-term effects on patients. Moreover, there are no obvious subjective symptoms at the early stage of uterine fibroids, and they are often detected by chance in physical examination. Nevertheless, the health examination rate of urban and rural residents is low in China, so that patients fail to discover the disease as early as possible, and the treatment is delayed. Therefore, it is of great significance to study the risk factors for uterine fibroids for early detection of high-risk groups.

Obesity is a chronic metabolic disease caused by the interaction of various factors. The findings of the Report on the Nutrition and Chronic Disease Status of Chinese Residents (2015) show that the obesity rate among residents of 18 years and above was increased from $4.5 \%$ in 2002 to $9.6 \%$ in 2012, and the obesity rate among adult females in 2014 was $8.0 \%$ based on the data in the China Health and Family Planning Statistical Yearbook $2017(3,4)$. A study showed that obesity leads to changes in endocrine system function, affects the levels of blood sex hormones and alters the secrete modes and metabolic pathways of hormones and their roles in target organs (5). Furthermore, some current studies have proved that obesity is one of the risk factors for uterine fibroids. However, studies on the risk of uterine fibroids in women with central obesity, especially those with relatively large visceral fat area (VFA), are rare.

Therefore, in this study, a case-control study on patients with uterine fibroids admitted to hospital and healthy patients receiving physical examination in the same period was conducted, and the relationships of indicators including overweight and obesity with the incidence rate of uterine fibroids were explored, providing a theoretical basis for the prevention and control of uterine fibroids. 


\section{Patients and methods}

Research objects and grouping. Uterine fibroid group included patients with uterine fibroids hospitalized in The Department of Gynecology of the First Hospital of Hebei Medical University (Shijiazhuang, China) from April 2016 to April 2018. Many hospitalizations were recorded as one case. A total of 89 patients definitely diagnosed with uterine fibroids according to medical history, clinical manifestations, gynecological physical examination, ultrasound examination and postoperative pathology were enrolled.

Control group included a total of 81 healthy women without uterine fibroids undergoing physical examination in the physical examination center in the same period.

This study was approved by the Ethics Committee of the First Hospital of Hebei Medical University. Patients who participated in this research had complete clinical data. The signed informed consents were obtained from the patients or the guardians.

Inclusion criteria. i) Patients aged 18-65 years; ii) patients without communication disorders, signed the informed consent and voluntarily participated in the study; and iii) patients having received body composition analytical examination.

Exclusion criteria. i) Patients aged less than 18 years old or over 65 years old; ii) patients with communication disorders or unwilling to participate in this study; iii) those with cardiac pacemakers or other implanted electronic medical devices; iv) patients with severe liver and kidney dysfunction, malignant tumors, infectious diseases or chronic debilitating diseases; and v) patients undergoing hysterectomy or hysteromyomectomy (without recurrence).

\section{Research methods}

Survey of basic information. Unified questionnaires were used by trained professionals to investigate basic information including name, age, education level and detailed medical history.

Measurement of body height and weight. The height and weight of patients wearing light clothes were measured in the early morning, with standing barefoot on an automatic height and weight measuring device (InBody BSMS370) after they emptied the stomach and the bladder and defecated.

Determination of body composition. The body composition of patients was measured using a body composition analyzer (InBody S10). The principle of direct segment multi-frequency bioelectrical impedance analysis (DSM-BIA) method was utilized to perform 30 electrical impedance measurements on 5 segments (the right upper and the left lower limbs, the trunk, and the right and the left lower limbs) through 6 different frequencies $(1,5,50,250,500$ and 1,000 $\mathrm{KHZ}$ ). Before measurement, patients were asked to remove accessories, and the places where electrodes were attached were wiped with alcohol, followed by measurement according to the operating instructions. After measurement, the following data were obtained: VFA, fat free mass, body fat content, body fat percentage, body mass index (BMI), upper arm circumference, upper arm muscle circumference and waist circumference.

Evaluation criteria. i) Grouping by VFA: Central obesity group $\left(\geq 100 \mathrm{~cm}^{2}\right)$ and non-central obesity group $\left(<100 \mathrm{~cm}^{2}\right)$; ii) Grouping by BMI: According to Criteria of Weight for
Adults (WS/T428-2013) (6) (a standard for health industry in the People's Republic of China), adults ( $>18$ years) are divided into underweight group (BMI $<18.5 \mathrm{~kg} / \mathrm{m}^{2}$ ), normal weight group ( $\geq 18.5 \mathrm{BMI}<24.0 \mathrm{~kg} / \mathrm{m}^{2}$ ), overweight group $\left(\geq 24.0 \mathrm{BMI}<28.0 \mathrm{~kg} / \mathrm{m}^{2}\right)$ and obesity group $\left(\mathrm{BMI} \geq 28.0 \mathrm{~kg} / \mathrm{m}^{2}\right)$; iii) grouping by waist circumference: according to Criteria of Weight for Adults (WS/T428-2013) (6) (a standard for health industry in the People's Republic of China), central pre-obesity: $\geq 80 \mathrm{~cm}$ female waist circumference $<85 \mathrm{~cm}$, and central obesity: Female waist circumference $\geq 85 \mathrm{~cm}$; iv) grouping by waist-height ratio: waist-height ratio is the ratio of waist circumference to height. It introduces height on the basis of waist circumference, effectively avoiding the height-induced bias in evaluating central obesity via waist circumference. It is an ideal index evaluating central obesity, and generally, waist-height ratio $\geq 0.5$ is the cut-off value in evaluating obesity based on waist-height ratio $(7,8)$; v) grouping by waist-hip ratio: waist-hip ratio is the ratio of waist circumference to hip circumference. According to the waist-hip ratio: waist-hip ratio $<0.85$ is normal somatotype, and waist-hip ratio $\geq 0.85$ is central obesity (9); and vi) grouping by body fat percentage: body fat percentage refers to the percentage of body fat content of the total body weight. According to the criteria for body fat content, people are divided into the following categories based on the fat content: i) underweight: body fat percentage $<17 \%$; ii) normal weight: $\geq 17 \%$ body fat percentage $<24 \%$; iii) overweight: $\geq 24 \%$ body fat percentage $<30 \%$; and iv) obesity: body fat percentage $\geq 30 \%$ (10).

Statistical analysis. SPSS 23.0 (IBM Corp., Armonk, NY, USA) was utilized for statistical analysis. Measurement data in accordance with normality were expressed as mean \pm standard deviation, and those not conforming to normality were expressed as median and quartile. Enumeration data were expressed as frequency. Independent sample t-test or rank sum test was applied for comparisons between two groups. Comparisons of indicators of overweight and obesity in patients with uterine fibroids among groups by age by one-way ANOVA or Kruskal-Wallis $\mathrm{H}$ test. Rank transformation procedures and Least Significant Difference as a post hoc test. $\chi^{2}$ test was used for calculation of odds ratio (OR) value and analysis of enumeration data. Logistic regression was used tofurther analyze the associations of overweight and obesity with the incidence rate of uterine fibroids. Spearman correlation was employed for correlation analysis. For all statistical analyses, two-sided test was performed, $\alpha=0.05$ was used as the test level, and $\mathrm{P} \leq 0.05$ was considered to indicate a statistically significant difference.

\section{Results}

Comparisons of general data. A total of 89 patients with uterine fibroids, whose average age was 44.22 \pm .19 years, were enrolled in this study, of which \% had no exercise habits, and $64.0 \%$ liked to eat sweets. A total of 81 patients with an average age of $44.00(32.00,50.50)$ years were selected as control group. There were no statistically significant differences in age and height between the two groups, and the data were comparable. 
Table I. General data.

\begin{tabular}{|c|c|c|c|c|}
\hline Indicator & Uterine fibroid group $(\mathrm{n}=89$ ) & Control group $(\mathrm{n}=81)$ & Statistic & P-value \\
\hline Age (years) & $44.22 \pm 7.19$ & $44.00(32.00,50.50)$ & $\mathrm{Z}=-1.466$ & 0.143 \\
\hline Height $(\mathrm{cm})$ & $157.48 \pm 6.03$ & $158.89 \pm 5.24$ & $t=-1.618$ & 0.108 \\
\hline Fat free mass $(\mathrm{kg})$ & $40.70(38.60,45.80)$ & $41.40(38.00,44.10)$ & $\mathrm{Z}=-0.378$ & 0.706 \\
\hline Weight (kg) & $61.60(56.50,68.50)$ & $58.65 \pm 7.72$ & $Z=-3.200$ & 0.001 \\
\hline $\operatorname{BMI}\left(\mathrm{kg} / \mathrm{m}^{2}\right)$ & $25.30(22.95,27.80)$ & $23.25 \pm 3.01$ & $Z=-4.283$ & $<0.05$ \\
\hline Body fat content $(\mathrm{kg})$ & $21.80(16.75,25.60)$ & $17.52 \pm 5.36$ & $\mathrm{Z}=-4.250$ & $<0.05$ \\
\hline Body fat percentage (\%) & $33.89 \pm 5.87$ & $30.60(24.05,34.85)$ & $Z=-4.062$ & $<0.05$ \\
\hline VFA $\left(\mathrm{cm}^{2}\right)$ & $105.10(73.35,129.45)$ & $74.10(55.95,99.90)$ & $Z=-4.590$ & $<0.05$ \\
\hline Waist circumference $(\mathrm{cm})$ & $85.50(79.65,94.65)$ & $79.20(73.75,84.15)$ & $Z=-5.081$ & $<0.05$ \\
\hline Waist-height ratio & $0.55 \pm 0.06$ & $0.50(0.46,0.54)$ & $\mathrm{Z}=-5.480$ & $<0.05$ \\
\hline Waist-hip ratio & $0.91 \pm 0.06$ & $0.85(0.82,0.89)$ & $Z=-6.203$ & $<0.05$ \\
\hline Upper arm circumference $(\mathrm{cm})$ & $30.80(28.75,33.30)$ & $28.82 \pm 2.42$ & $Z=-5.242$ & $<0.05$ \\
\hline Upper arm muscle circumference $(\mathrm{cm})$ & $26.36 \pm 2.18$ & $24.63 \pm 1.79$ & $\mathrm{t}=5.669$ & $<0.05$ \\
\hline Right upper limb muscle (kg) & $2.14(1.92,2.45)$ & $2.02(1.86,2.23)$ & $Z=-2.961$ & 0.003 \\
\hline Left upper limb muscle $(\mathrm{kg})$ & $2.19 \pm 0.38$ & $1.96(1.83,2.18)$ & $Z=-3.189$ & 0.001 \\
\hline Trunk muscle $(\mathrm{kg})$ & $19.28 \pm 2.41$ & $18.30(17.10,19.30)$ & $Z=-2.826$ & 0.005 \\
\hline Right lower limb muscle (kg) & $6.22 \pm 0.96$ & $6.27 \pm 0.93$ & $t=-0.339$ & 0.735 \\
\hline Left lower limb muscle $(\mathrm{kg})$ & $6.21 \pm 0.93$ & $6.24(5.61,6.67)$ & $Z=-0.334$ & 0.738 \\
\hline Skeletal muscle (kg) & $22.79 \pm 3.12$ & $22.30(20.35,24.00)$ & $Z=-0.696$ & 0.487 \\
\hline Muscle mass (kg) & $39.24 \pm 4.90$ & $38.70 \pm 4.54$ & $\mathrm{t}=0.749$ & 0.455 \\
\hline Bone mineral content $(\mathrm{kg})$ & $2.33(2.17,2.55)$ & $2.42 \pm 0.32$ & $Z=-0.991$ & 0.322 \\
\hline Protein content (kg) & $8.21 \pm 1.02$ & $8.05 \pm 0.94$ & $\mathrm{t}=1.055$ & 0.293 \\
\hline Inorganic salt $(\mathrm{kg})$ & $2.88 \pm 0.42$ & $2.92 \pm 0.38$ & $\mathrm{t}=-0.651$ & 0.516 \\
\hline Total body water (l) & $29.80(28.30,33.60)$ & $30.15 \pm 3.55$ & $Z=-0.816$ & 0.415 \\
\hline Extracellular water (1) & $11.50(10.75,12.80)$ & $11.50(10.60,12.20)$ & $Z=-0.755$ & 0.450 \\
\hline Intracellular water (1) & $19.02 \pm 2.38$ & $18.64 \pm 2.18$ & $\mathrm{t}=1.072$ & 0.285 \\
\hline Resting metabolic rate (kcal) & $1274.51 \pm 114.19$ & $1258.37 \pm 104.46$ & $\mathrm{t}=0.958$ & 0.339 \\
\hline Body cell count $(\mathrm{kg})$ & $27.23 \pm 3.41$ & $26.69 \pm 3.12$ & $\mathrm{t}=1.083$ & 0.280 \\
\hline Water matching ratio $(\%)$ & $73.40(73.30,73.60)$ & $73.33 \pm 0.27$ & $\mathrm{Z}=-3.214$ & 0.001 \\
\hline General phase angle & $5.52 \pm 0.65$ & $5.40(5.00,5.80)$ & $Z=-0.923$ & 0.356 \\
\hline
\end{tabular}

BMI, body mass index; VFA, visceral fat area.

Table II. Comparisons of indicators of overweight and obesity in patients with uterine fibroids among groups by age by one-way ANOVA or Kruskal-Wallis H test.

\begin{tabular}{lccccc}
\hline Indicator & $\leq 40$ years & $41-50$ years & $\geq 51$ years & Statistic & P-value \\
\hline BMI $\left(\mathrm{kg} / \mathrm{m}^{2}\right)$ & $23.95(21.83,26.83)^{\mathrm{a}}$ & $25.68 \pm 3.23$ & $27.03 \pm 3.11$ & $\mathrm{H}=6.431$ & 0.040 \\
Body fat percentage $(\%)$ & $32.53 \pm 6.77$ & $33.82 \pm 5.34$ & $36.15 \pm 5.38$ & $\mathrm{~F}=2.005$ & 0.141 \\
VFA $\left(\mathrm{cm}^{2}\right)$ & $75.85(63.65,125.33)^{\mathrm{a}}$ & $102.75(79.83,122.50)^{\mathrm{b}}$ & $123.60 \pm 35.15$ & $\mathrm{H}=6.235$ & 0.044 \\
Waist circumference $(\mathrm{cm})$ & $81.25(77.98,91.93)^{\mathrm{a}}$ & $86.67 \pm 9.09$ & $91.32 \pm 8.39$ & $\mathrm{H}=6.307$ & 0.043 \\
Waist-height ratio & $0.52(0.49,0.59)$ & $0.55 \pm 0.05$ & $0.58 \pm 0.05$ & $\mathrm{H}=4.982$ & 0.083 \\
Waist-hip ratio & $0.90(0.86,0.93)$ & $0.91 \pm 0.05$ & $0.92 \pm 0.06$ & $\mathrm{H}=1.805$ & 0.406
\end{tabular}

BMI, body mass index; VFA, visceral fat area. ${ }^{a} \mathrm{P}<0.05$, compared to $\geq 51$ years group; ${ }^{\mathrm{b}} \mathrm{P}<0.05$, compared to $\geq 51$ years group.

According to Table I, the difference in the weight between the two groups was statistically significant, but no statistically significant differences were detected in the fat free mass, muscle mass and skeletal muscle, indicating that the difference in the weight between the two groups is mainly caused by fat level. Further studies found that uterine fibroid 
Table III. Correlation analyses of overweight and obesity indicators with age.

\begin{tabular}{lcc}
\hline Indicator & $\mathrm{r}$ & P-value \\
\hline BMI $\left(\mathrm{kg} / \mathrm{m}^{2}\right)$ & 0.212 & 0.046 \\
Body fat percentage $(\%)$ & 0.168 & 0.116 \\
Waist circumference $(\mathrm{cm})$ & 0.262 & 0.013 \\
Waist-height ratio & 0.168 & 0.116 \\
Waist-hip ratio & 0.058 & 0.591 \\
VFA $\left(\mathrm{cm}^{2}\right)$ & 0.248 & 0.019 \\
\hline
\end{tabular}

BMI, body mass index; VFA, visceral fat area.

group had significantly elevated levels of upper limb muscles and trunk muscles and an overtly decreased lower limb muscle level in comparison with control group, suggesting that most patients with uterine fibroids have declined lower limb muscle levels. The waist circumference and VFA in uterine fibroid group were higher than those in control group, and the differences were statistically significant, implying that most patients with uterine fibroids suffer from central obesity.

Tables II and III revealed that the BMI, VFA and waist circumference of patients with uterine fibroids were gradually elevated with increasing age, showing statistically significant differences.

Relationships of overweight and obesity indicators with the incidence rate of uterine fibroids. The VFA, BMI, body fat percentage, waist circumference, waist-height ratio and waist-hip ratio are common indicators evaluating overweight and obesity. Table IV showed that overweight and obesity were risk factors for uterine fibroids. The OR [95\% confidence interval (CI)] of the waist-hip ratio was 7.707 (3.501, 16.965), which was significantly increased compared with that of other indicators. Logistic regression was used to further analyze the associations of overweight and obesity with the incidence rate of uterine fibroids, and it was found that the risk of uterine fibroids was increased with enhanced VFA, BMI, waist circumference, waist-height ratio and waist-hip ratio. Age is a risk factor for uterine fibroids (11). Therefore, age was adjusted as a confounding factor, and the analysis results were not changed. The specific results are shown in Tables V-X.

Correlations of overweight and obesity indicators with the size of uterine fibroids. Spearman correlation analysis was carried out to study the correlations of the indicators of overweight and obesity with the size of uterine fibroids, and the results showed that the VFA and body fat percentage were related to the size of uterine fibroids, but the correlations were relatively weak (Table XI).

Relations of nutrition-related indicators with uterine fibroids. Uterine fibroid group had lower bone mineral content and inorganic salt in comparison with control group, but the differences were not statistically significant (Table I).

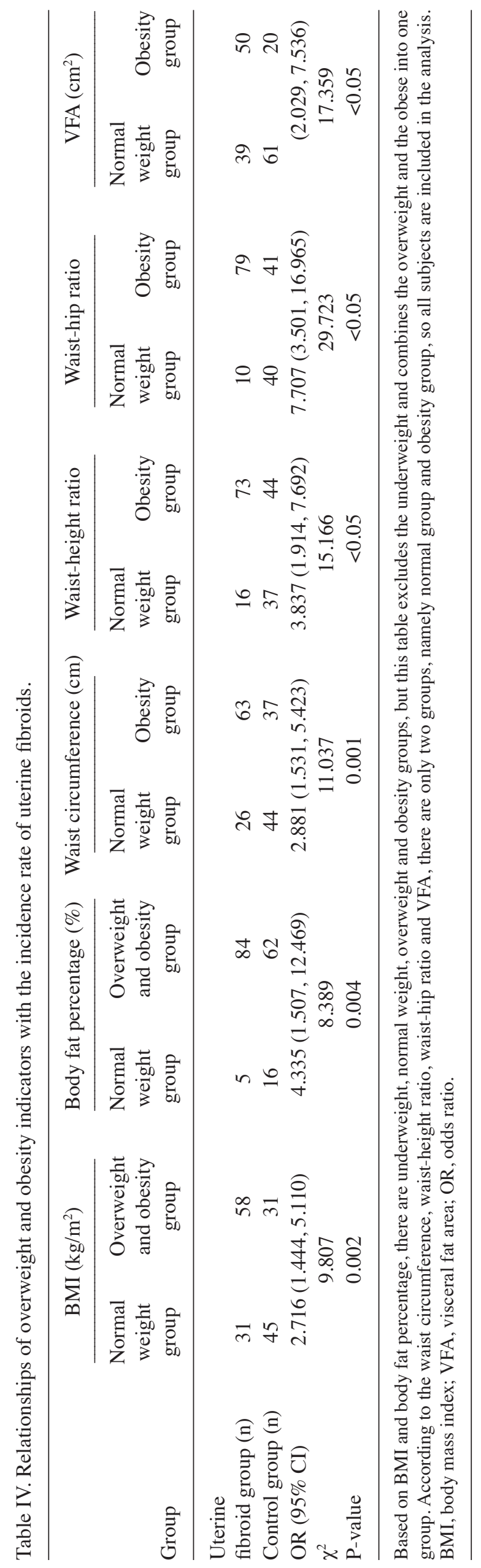


Table V. Logistic regression analysis of the relationship between BMI and the incidence rate of uterine fibroids.

\begin{tabular}{lcccc}
\hline & \multicolumn{3}{c}{ BMI $\left(\mathrm{kg} / \mathrm{m}^{2}\right)$} \\
\cline { 2 - 5 } Index & $>22.08(\mathrm{Q} 1)$ & $22.08-24.15(\mathrm{Q} 2)$ & $24.16-26.73(\mathrm{Q} 3)$ & $<26.73(\mathrm{Q} 4)$ \\
\hline Uterine fibroid group $(\mathrm{n})$ & 14 & 19 & 25 & 31 \\
Control group (n) & 28 & 24 & 18 & 11 \\
Univariate analysis OR (95\% CI) & 1 & $1.583(0.657,3.816)$ & $2.778(1.149,6.713)$ & $5.636(2.200,14.438)$ \\
P-value & 1 & 0.306 & 0.023 & $<0.05$ \\
OR (95\% CI) of adjusted age & & $1.499(0.606,3.707)$ & $2.564(1.006,6.533)$ & $5.167(1.904,14.023)$ \\
P-value & & 0.381 & 0.049 & 0.001 \\
\hline
\end{tabular}

BMI, body mass index; OR, odds ratio; CI, confidence interval.

Table VI. Logistic regression analysis of the relation between body fat percentage and the incidence rate of uterine fibroids.

\begin{tabular}{lcccc}
\hline & \multicolumn{4}{c}{ Body fat percentage $(\%)$} \\
\cline { 2 - 5 } Index & $>26.98(\mathrm{Q} 1)$ & $26.98-32.64(\mathrm{Q} 2)$ & $32.65-36.00(\mathrm{Q} 3)$ & $<36.00(\mathrm{Q} 4)$ \\
\hline Uterine fibroid group $(\mathrm{n})$ & 9 & 27 & 24 & 29 \\
Control group (n) & 33 & 16 & 20 & 12 \\
Univariate analysis OR (95\% CI) & 1 & $6.187(2.364,16.194)$ & $4.400(1.708,11.334)$ & $8.861(3.267,24.037)$ \\
P-value & 1 & $<.213(2.286,16,889)$ & $4.420(1.640,11,913)$ & $8.914(3.056,26,003)$ \\
OR (95\% CI) of adjusted age & & $<0.05$ & 0.003 & $<0.05$ \\
P-value & & &
\end{tabular}

OR, odds ratio; CI, confidence interval.

Table VII. Logistic regression analysis of the association between waist circumference and the incidence rate of uterine fibroids.

\begin{tabular}{lcccc}
\hline & \multicolumn{4}{c}{ Waist circumference $(\mathrm{cm})$} \\
\cline { 2 - 5 } Index & $>77.25(\mathrm{Q} 1)$ & $77.25-82.09(\mathrm{Q} 2)$ & $82.10-89.93(\mathrm{Q} 3)$ & $<89.93(\mathrm{Q} 4)$ \\
\hline Uterine fibroid (n) & 11 & 19 & 27 & 32 \\
Control (n) & 31 & 24 & 16 & 10 \\
Univariate analysis OR (95\% CI) & 1 & $2.231(0.894,5.565)$ & $4.756(1.886,11.992)$ & $9.018(3.356,24.236)$ \\
P-value & 1 & $2.273(0.887,5.821)$ & $4.877(1.843,12.911)$ & $9.344(3.185,27.411)$ \\
OR (95\% CI) of adjusted age & & 0.087 & 0.001 & $<0.05$ \\
P-value & & &
\end{tabular}

OR, odds ratio; CI, confidence interval.

\section{Discussion}

Currently, studies have suggested that the conversion of estrone to estradiol in uterine fibroids is significantly lower than that in normal muscle tissues, and the concentration of estrogen receptors in fibroids is clearly higher than that in peripheral muscle tissues. Therefore, the pathogenesis of uterine fibroids may be related to sex hormone levels (12). Obesity is able to cause metabolic disorders, making local tissues to form an abnormally high estrogen environment. The mechanism includes: i) androstenedione secreted by adrenal glands can be converted into estrone by aromatase in adipose tissues, and the level of estrone in plasma is increased with elevated adipose tissues and strengthened conversion ability, thus leading to persistent estrogen effect; and ii) obesity leads to the lack of periodic regulation of progesterone, so that the endometrium is in an environment of over stimulation from no estrogen antagonistic progesterone for a long time $(11,13)$. Uterine 
Table VIII. Logistic regression analysis of the correlation between waist-height ratio and the incidence rate of uterine fibroids.

\begin{tabular}{lcccc}
\hline & \multicolumn{4}{c}{ Waist-height ratio } \\
\cline { 2 - 5 } Index & $>0.48(\mathrm{Q} 1)$ & $0.48-0.52(\mathrm{Q} 2)$ & $0.53-0.57(\mathrm{Q} 3)$ & $<0.57(\mathrm{Q} 4)$ \\
\hline Uterine fibroid group (n) & 4 & 26 & 27 & 32 \\
Control group (n) & 29 & 25 & 20 & 7 \\
Univariate analysis OR (95\% CI) & 1 & $7.540(2.315,24.557)$ & $9.787(2.964,32.322)$ & $33.143(8.789,124.973)$ \\
P-value & 1 & $8.900(2.594,30.535)$ & $12.705(3.432,47.073)$ & $44.511(10.311,192.139)$ \\
OR (95\% CI) of adjusted age & & 0.001 & $<0.05$ & $<0.05$ \\
P-value & & & $<0.05$ & $<$ \\
\hline
\end{tabular}

OR, odds ratio; CI, confidence interval.

Table IX. Logistic regression analysis of the relationship between waist-hip ratio and the incidence rate of uterine fibroids.

\begin{tabular}{lcccc}
\hline & \multicolumn{4}{c}{ Waist-hip ratio } \\
\cline { 2 - 5 } Index & $>0.84(\mathrm{Q} 1)$ & $0.84-0.87(\mathrm{Q} 2)$ & $0.88-0.92(\mathrm{Q} 3)$ & $<0.92(\mathrm{Q} 4)$ \\
\hline Uterine fibroid group (n) & 7 & 19 & 31 & 32 \\
Control group (n) & 33 & 22 & 19 & 7 \\
Univariate analysis OR (95\% CI) & 1 & $4.071(1.467,11.300)$ & $7.692(2.842,20.817)$ & $21.551(6.790,68.403)$ \\
P-value & & 0.007 & $<0.05$ & $<0.05$ \\
OR (95\% CI) of adjusted age & 1 & $3.993(1.434,11.119)$ & $7.494(2.755,20.390)$ & $20.357(6.275,66.045)$ \\
P-value & & 0.008 & $<0.05$ & $<0.05$
\end{tabular}

OR, odds ratio; CI, confidence interval.

Table X. Logistic regression analysis of the relationship between VFA and the incidence rate of uterine fibroids.

\begin{tabular}{lcccc}
\hline & \multicolumn{3}{c}{ VFA $\left(\mathrm{cm}^{2}\right)$} \\
\cline { 2 - 5 } Index & $>61.35(\mathrm{Q} 1)$ & $61.35-90.24(\mathrm{Q} 2)$ & $90.25-114.27(\mathrm{Q} 3)$ & $<114.27(\mathrm{Q} 4)$ \\
\hline Uterine fibroid group (n) & 13 & 21 & 21 & 34 \\
Control group (n) & 29 & 22 & 8 \\
Univariate analysis OR (95\% CI) & 1 & $2.129(0.878,5.166)$ & $2.129(0.878,5.166)$ & $9.481(3.451,26.044)$ \\
P-value & 1 & $2.057(0.822,5,147)$ & $2.026(0.784,5,236)$ & $8.972(3.055,26,343)$ \\
OR (95\% CI) of adjusted age & & 0.123 & 0.145 & $<0.05$ \\
P-value & & & $<0.05$ & $<$ \\
\hline
\end{tabular}

VFA, visceral fat area, OR, odds ratio; CI, confidence interval.

Table XI. Correlation analysis of overweight and obesity indicators with the size of uterine fibroids.

\begin{tabular}{lcc}
\hline Indicator & $\mathrm{r}$ & P-value \\
\hline Body mass index $\left(\mathrm{kg} / \mathrm{m}^{2}\right)$ & -0.194 & 0.069 \\
Body fat percentage $(\%)$ & -0.222 & 0.036 \\
Waist circumference $(\mathrm{cm})$ & -0.178 & 0.095 \\
Waist-height ratio & -0.178 & 0.095 \\
Waist-hip ratio & -0.072 & 0.500 \\
Visceral fat area $\left(\mathrm{cm}^{2}\right)$ & -0.223 & 0.036 \\
\hline
\end{tabular}

fibroids may be induced in an abnormally high estrogen environment. Therefore, obesity may be one of the risk factors for uterine fibroids.

A case-control study manifested that the BMI, waist circumference, hip circumference, waist-height ratio, body fat content and body fat percentage are positively correlated with the development of uterine fibroids, women with high BMI and waist-hip ratio have the highest risk of uterine fibroids, those with the body fat percentage of more than $30 \%$ also have relatively high risk of uterine fibroids, and uterine fibroids may have relationships with overweight and 
central obesity (14), which are consistent with some findings of this study.

Visceral fat acts as hormone-active tissues and can cause uterine fibroids by increasing the production of inflammatory mediators. Ciavattini et al (15) measured the extra-peritoneal fat thickness (PFT) and subcutaneous fat of patients with uterine fibroids and controls via echography and discovered that the PFT is independently correlated with the development of uterine fibroids $(\mathrm{P}<0.0001)$, and the ROC curve analysis confirmed that the PFT $=6.7 \mathrm{~mm}$ can be used as a cut-off value for determining the development of uterine fibroids. The results of this study also showed that the VFA was a risk factor for uterine fibroids, the risk of uterine fibroids was elevated with the increase in the VFA, and the VFA was correlated with the size of uterine fibroids although the correlation was relatively weak. Besides, the estrogen in perimenopausal patients was decreased with increasing age, which might lead to visceral fat accumulation. Therefore, perimenopausal women should pay attention to diet and exercise to maintain healthy weight.

In conclusion, obesity is one of the risk factors for uterine fibroids, and increased body fat (especially abdominal visceral fat) is capable of raising the risk of uterine fibroids. The waist-hip ratio of perimenopausal women can often be measured as an indicator screening high-risk groups of uterine fibroids. Meanwhile, providing nutrition guidance and changing diet and exercise habits are important measures to prevent the development of uterine fibroids.

\section{Acknowledgements}

Not applicable.

\section{Funding}

This worked was funded by Project of health commission of Hebei province (No. 20170518).

\section{Availability of data and materials}

The datasets used and/or analyzed during the current study are available from the corresponding author on reasonable request.

\section{Authors' contributions}

KS wrote the manuscript. KS and YX were responsible for determination of body composition. NZ and ZL collected and analyzed general information of patients. All authors read and approved the final manuscript.

\section{Ethics approval and consent to participate}

This study was approved by the Ethics Committee of the First Hospital of Hebei Medical University (Shijiazhuang, China). Patients who participated in this research had complete clinical data. The signed informed consents were obtained from the patients or the guardians.

\section{Patient consent for publication}

Not applicable.

\section{Competing interests}

The authors declare that they have no competing interests.

\section{References}

1. Moore AB, Flake GP, Swartz CD, Heartwell G, Cousins D, Haseman JK, Kissling GE, Sidawy MK and Dixon D: Association of race, age and body mass index with gross pathology of uterine fibroids. J Reprod Med 53: 90-96, 2008.

2. Van Voorhis B: A 41-year-old woman with menorrhagia, anemia, and fibroids: Review of treatment of uterine fibroids. JAMA 301: 82-93, 2009.

3. Davy BM, Zoellner JM, Waters CN, Bailey AN and Hill JL: Associations among chronic disease status, participation in federal nutrition programs, food insecurity, and sugar-sweetened beverage and water intake among residents of a health-disparate region. J Nutr Educ Behav 47: 196-205, 2015.

4. Li H, Liu K, Gu J, Zhang Y, Qiao Y and Sun X: The development and impact of primary health care in China from 1949 to 2015: a focused review. Int J Health Plann Manag 32: 339-350, 2017.

5. Mayes JS and Watson GH: Direct effects of sex steroid hormones on adipose tissues and obesity. Obes Rev 5: 197-216, 2004.

6. The People's Republic of China National Health and Family Planning Commission: Criteria of Weight for Adults. China Zhijian Publishing House, Beijing, 2013 (In Chinese).

7. Shao J, Yu L, Shen X, Li D and Wang K: Waist-to-height ratio, an optimal predictor for obesity and metabolic syndrome in Chinese adults. J Nutr Health Aging 14: 782-785, 2010.

8. Zeng Q, He Y, Dong S, Zhao X, Chen Z, Song Z, Chang G, Yang F and Wang Y: Optimal cut-off values of BMI, waist circumference and waist:height ratio for defining obesity in Chinese adults. Br J Nutr 112: 1735-1744, 2014.

9. Döring N, Mayer S, Rasmussen F and Sonntag D: Economic evaluation of obesity prevention in early childhood: methods, limitations and recommendations. Int J Environ Res Public Health 13: 13, 2016.

10. Infante JR, Reyes C, Ramos M, Rayo JI, Lorente R, Serrano J, Domínguez ML, García L, Durán C and Sánchez R: The usefulness of densitometry as a method of assessing the nutritional status of athletes. Comparison with body mass index. Rev Esp Med Nucl Imagen Mol 32: 281-285, 2013.

11. Zimmermann A, Bernuit D, Gerlinger C, Schaefers M and Geppert K: Prevalence, symptoms and management of uterine fibroids: An international internet-based survey of 21,746 women. BMC Womens Health 12: 6, 2012.

12. Wong JY, Gold EB, Johnson WO and Lee JS: Circulating sex hormones and risk of uterine fibroids: Study of Women's Health Across the Nation (SWAN). J Clin Endocrinol Metab 101: 123-130, 2016.

13. Curti ML, Jacob P, Borges MC, Rogero MM and Ferreira SR: Studies of gene variants related to inflammation, oxidative stress, dyslipidemia, and obesity: implications for a nutrigenetic approach. J Obes 2011: 497401, 2011.

14. Chen CR, Buck GM, Courey NG, Perez KM and Wactawski-Wende J: Risk factors for uterine fibroids among women undergoing tubal sterilization. Am J Epidemiol 153: 20-26, 2001.

15. Ciavattini A, Di Giuseppe J, Stortoni P, Montik N, Giannubilo SR, Litta P, Islam MS, Tranquilli AL, Reis FM and Ciarmela P: Uterine fibroids: pathogenesis and interactions with endometrium and endomyometrial junction. Obstet Gynecol Int 2013: 173184, 2013.

This work is licensed under a Creative Commons Attribution-NonCommercial-NoDerivatives 4.0 International (CC BY-NC-ND 4.0) License. 